\title{
The Contribution of Known Familial Cardiovascular Disease Genes to Sudden Cardiac Death in Patients Undergoing Hemodialysis
}

\author{
Tae-Hwi Schwantes-An ${ }^{a}$ Matteo Vatta ${ }^{a}$ Marco Abreu ${ }^{a}$ Leah Wetherilla \\ Howard J. Edenberg ${ }^{a, b}$ Tatiana M. Foroud ${ }^{a}$ Glenn M. Chertow ${ }^{c}$ \\ Sharon M. Moed, e \\ aDepartment of Medical and Molecular Genetics, Indiana University School of Medicine, Indianapolis, IN, USA; \\ ${ }^{b}$ Department of Biochemistry and Molecular Biology, Indiana University School of Medicine, Indianapolis, IN, USA; \\ 'Division of Nephrology, Department of Medicine, Stanford University, Stanford, CA, USA; dDivision of Nephrology \\ and Hypertension, Department of Medicine, Indiana University School of Medicine, Indianapolis, IN, USA; \\ eDepartment of Medicine, Roudebush Veterans Administration Medical Center, Indianapolis, IN, USA
}

\section{Keywords}

Cardiovascular disease · Sudden cardiac death · Arrhythmia · Dialysis · Kidney

\begin{abstract}
Introduction: Patients with chronic kidney disease experience high rates of cardiovascular mortality and morbidity. When kidney disease progresses to the need for dialysis, sudden cardiac death (SCD) accounts for $25-35 \%$ of all cardiovascular deaths. The objective was to determine if rare genetic variants known to be associated with cardiovascular death in the general population are associated with SCD in patients undergoing hemodialysis. Methods: We performed a case-control study comparing 126 (37 African American [AfAn] and 89 European ancestry [EA]) SCD subjects and 107 controls (34 AfAn and $73 \mathrm{EA}$ ), matched for age, sex, self-reported race, dialysis duration ( $<2,2-5$ and $>5$ years), and the presence or absence of diabetes mellitus. To target the coding regions of genes previously reported to be associated with 15 inherited cardiac conditions (ICCS), we used the TruSight Cardio Kit (Illumina, San Diego, CA, USA) to capture the genetic regions of interest. In all, the kit targets $572-\mathrm{kb}$ regions that include the protein-coding regions and 40-bp $5^{\prime}$
\end{abstract}

karger@karger.com www.karger.com/crm

Karger $\stackrel{\text { ' }}{5}$
(C) 2021 The Author(s)

Published by S. Karger AG, Basel

This is an Open Access article licensed under the Creative Commons Attribution-NonCommercial-4.0 International License (CC BY-NC) (http://www.karger.com/Services/OpenAccessLicense), applicable to the online version of the article only. Usage and distribution for commercial purposes requires written permission. and $3^{\prime}$ end-flanking regions of 174 genes associated with the 15 ICCs. Using the sequence data, burden tests were conducted to identify genes with an increased number of variants among SCD cases compared to matched controls. $\boldsymbol{R e}$ sults: Eleven genes were associated with $\mathrm{SCD}$, but after correction for multiple testing, none of the 174 genes were identified as having more variants in the SCD cases than the matched controls, including previously identified genes. Secondary burden tests grouping variants based on diseases and gene function did not produce statistically significant associations. Discussion/Conclusions: We found no associations between genes known to be associated with ICCs and SCD in our sample of patients undergoing hemodialysis. This suggests that genetic causes are unlikely to be a major pathogenic factor in SCD in hemodialysis patients, although our sample size limits definitive conclusions.

(C) 2021 The Author(s)

Published by S. Karger AG, Basel

\section{Introduction}

Cardiovascular disease is the major cause of death in patients with chronic kidney disease (CKD). When patients have progressive $\mathrm{CKD}$ and require dialysis (end- 
stage kidney disease [ESKD]), cardiovascular manifestations change from a predominant atherosclerotic disease to a left ventricular hypertrophy and heart failure with preserved ejection fraction. In patients with ESKD, diastolic dysfunction, non-ST elevation myocardial infarction, and bradyarrhythmias are more common than systolic dysfunction, ST elevation infarction, and ventricular arrhythmias [1-3]. Sudden cardiac death (SCD) accounts for $25-35 \%$ of all cardiovascular deaths in patients with ESKD in both adjudicated clinical trials [4] and in association studies of large databases $[5,6]$. The dialysis procedure had been assumed to cause SCD due to rapid volume and electrolyte shifts. However, studies also show an increased risk of SCD with progressive CKD, often long before dialysis is required (reviewed in [7]), suggesting that CKD itself, and/or its attendant metabolic abnormalities, may be a risk factor for SCD. Importantly, a study of patients undergoing dialysis in the Fresenius Medical Care ESKD database identified pairs of genetically related family members who had suffered a fatal cardiac arrest/ arrhythmia, and phenotypically matched control pairs. The risk of cardiac arrest/arrhythmia was increased to 1.88 (95\% CI 1.25-2.84) for family members who did not cohabitate [8], suggesting a possible genetic cause of SCD.

A study of 47 patients with ESKD and a prolonged QT interval found 5 genes associated with arrhythmias; in the follow-up, 2 patients died of SCD [9]. We previously reported that 3 correlated single-nucleotide polymorphisms (SNPs) in the angiotensin-converting enzyme ( $A C E)$ gene were associated with lower rates of SCD in European Americans enrolled in the Effect of Cinacalcet on Cardiovascular Disease in Patients Undergoing Dialysis (EVOLVE) trial. One ACE SNP, rs4318, only found in the African American sample, was associated with a higher rate of SCD [10]. Thus, there is some evidence that genetics may be important in SCD in patients with ESKD. However, the association of rare genetic variants, especially those associated with SCD in the general population [11], has not been examined in patients on dialysis. Given the disproportionate frequency of SCD in ESKD, we examined these variants in a case-control study using DNA and adjudicated endpoints from the EVOLVE trial $[12,13]$.

\section{Materials and Methods}

\section{Participant Selection}

The EVOLVE trial randomized 3,883 patients with ESKD receiving hemodialysis thrice weekly with moderate to severe secondary hyperparathyroidism to either cinacalcet or placebo in addition to standard-of-care treatments which typically included phosphate binders and calcitriol (1,25-dihydroxy vitamin D) or other active vitamin D analogs. An independent clinical events committee adjudicated all primary and secondary endpoints, including SCD [13]. The primary endpoint of the EVOLVE trial was a composite of all-cause mortality, nonfatal myocardial infarction, hospitalization for unstable angina, heart failure, and peripheral vascular event. A post hoc analysis [4] found that $54 \%$ of all deaths in the EVOLVE trial were due to cardiovascular disease, and of those, nearly 1 in 4 (352 patients) was due to SCD, whereas only $4 \%$ of deaths were due to acute myocardial infarction. Of the 3,883 EVOLVE participants, 1,919 (49\%) consented to have DNA collected (confirmed internally at Amgen), and 1,852 samples were of adequate quality to be genotyped, and the project was approved by the Indiana University Institutional Review Board [14]. Of those samples with DNA, we identified 126 patients with SCD (37 African American [AfAn] and 89 European ancestry [EA] samples) and then selected 107 controls (34 AfAn and 73 EA) who were matched for age, sex, self-reported race, dialysis duration $(<2,2-5$ and $>5$ years), and the presence or absence of diabetes for sequencing.

\section{DNA Sequencing}

DNA was extracted from white cell pellets or peripheral blood obtained from the 233 participants (126 cases and 107 controls) who were chosen for sequencing. To target the coding regions of genes previously reported to be associated with 15 inherited cardiac conditions (ICCs: long QT syndrome [LQTS], short QT syndrome [SQTS], Brugada syndrome [BrS], catecholaminergic polymorphic ventricular tachycardia [CPVT], hypertrophic cardiomyopathy $[\mathrm{HCM}]$, dilated cardiomyopathy [DCM], arrhythmogenic right ventricular cardiomyopathy [ARVC], restrictive cardiomyopathy [RCM], left ventricular noncompaction [LVNC], Noonan syndrome, Marfan syndrome [MFS], Loeys-Dietz syndrome [LDS], familial aortic aneurysm, aortic valve disease, and familial hypercholesterolemia), we used the TruSight Cardio Kit (Illumina, San Diego, CA, USA) to capture the coding regions of the reported genes. In all, the kit targets $572-\mathrm{kb}$ regions that include the protein coding regions and 40-bp $5^{\prime}$ and $3^{\prime}$ end-flanking regions of 174 genes associated with the 15 ICCs (genetic details of the genes in the supplement of [15]). Sequencing was conducted at the IU Molecular Genetics Diagnostic Laboratory (IUMGL) at the Indiana University School of Medicine (IUSM) using MiSeq (Illumina, San Diego, CA, USA) following the manufacturer's procedures.

\section{Sequence Data Processing}

We processed sequence data using the in-house NextGen sequence data pipeline that uses Genome Analysis Tool Kit version 4 [16] following the best practices [17] guideline. In brief, reads were first mapped to human reference genome build 38 using BWA [18]. We then sorted, de-duplicated, and recalibrated base quality scores from mapped reads using Genome Analysis Tool Kit version 4. Using HaplotypeCaller, we identified variants in each individual separately, resulting in a gvcf file for each EVOLVE participant. Those gvcf files were then combined for group-level variant calling. The resulting file was a set of SNPs and insertion/deletions. Due to the limited number of samples and limited size of sequenced regions, we filtered variants based on the parameters (below), instead of the more typical variant quality score recalibration procedure in GATK. We required the following: (a) quality by depth $\geq 2.0$, (b) Fisher strand $\leq 60.0$, (c) root-mean-square of the 
Table 1. Baseline demographics and laboratory values

\begin{tabular}{|c|c|c|c|}
\hline Cinacalcet use, $n(\%)$ & $60(48)$ & $52(49)$ & $1,948(50)$ \\
\hline Age, years, mean (SD) & $59.7(11.5)$ & $59.6(11.8)$ & $54.4(14.4)^{+}$ \\
\hline Sex (female), $n(\%)$ & $51(40)$ & $44(41)$ & $1,578(41)$ \\
\hline \multicolumn{4}{|l|}{ Ethnicity (self-reported), $n(\%)$} \\
\hline Other & $0(0)$ & $0(0)$ & $806(21)$ \\
\hline \multicolumn{4}{|l|}{ Dialysis vintage, $n(\%)$} \\
\hline$<2$ years & $36(29)$ & $31(29)$ & $1,098(28)$ \\
\hline 2 to $<5$ years & $45(36)$ & $37(35)$ & $1,285(33)$ \\
\hline$\geq 5$ years & $45(36)$ & $39(36)$ & $1,499(39)$ \\
\hline Current or previous smoker, $n(\%)$ & $58(46)$ & $53(50)$ & $1,696(44)$ \\
\hline Hypertension, $n(\%)$ & $118(94)$ & $101(94)$ & $3,577(92)$ \\
\hline Coronary artery bypass graft, $n(\%)$ & $15(12)$ & $11(10)$ & $289(7)$ \\
\hline Myocardial infarction, $n(\%)$ & $19(15)$ & $17(16)$ & $483(12)$ \\
\hline Heart failure, $n(\%)$ & $44(35)$ & $36(34)$ & $906(23)$ \\
\hline Stroke, $n(\%)$ & $14(11)$ & $13(12)$ & $355(9)$ \\
\hline Peripheral arterial disease, $n(\%)$ & $28(22)$ & $27(25)$ & $635(16)$ \\
\hline Amputation, $n(\%)$ & $8(6)$ & $13(12)$ & $250(6)$ \\
\hline \multicolumn{4}{|l|}{ Baseline drug use, $n$ (\%) } \\
\hline Calcium-based phosphate binders & $70(56)$ & $49(46)$ & $2,062(53)$ \\
\hline Baseline vitamin $\mathrm{D}$ & $75(60)$ & $78(73)$ & $2,310(59)$ \\
\hline \multicolumn{4}{|l|}{ Baseline laboratory values } \\
\hline Corrected Ca, mean (SD), mg/dL & $9.8(0.7)$ & $9.9(0.7)$ & $9.8(0.7)$ \\
\hline
\end{tabular}

${ }^{+} p<0.0018$ SCD versus entire EVOLVE cohort.

mapping quality of the reads across all samples $\geq 40.0$, (d) mapping quality rank sum test statistic $\geq-12.5$, (e) read position rank sum test statistic $\geq 8.0$, and (f) strand odds ratio $\leq 3.0$ for SNPs in the vcf file. For the insertion/deletions, we required the following: (a) quality by depth $\geq 2.0$, (b) Fisher strand $\leq 200.0$, (c) read position rank sum test statistic $\geq 20.0$, and (d) strand odds ratio $\leq 3.0$. After filtering, we annotated all variants that passed QC using ANNOVAR [19].

Variant Selection for Analysis

Variants passing QC, as described earlier, were filtered based on their annotated functions and minor allele frequencies (MAFs) from gnomAD [20] prior to burden testing. First, we kept those SNPs that were predicted to change the amino acid sequence (coding change) based on the annotated functions, including frame- shift insertion, frameshift deletion, nonsynonymous SNV, stopgain, and stoploss. For those variants predicted to be functional, we removed those with MAFs $\geq 5 \%$ based on reported populationlevel frequencies from gnomAD non-Finnish European and African samples. After filtering for predicted function (amino acid coding changing) and population-based MAFs (MAF <5\%), AfAn samples had 1,514 variants and EA samples had 1,610 variants to be used in the analyses in 153 genes.

\section{Statistical Analysis}

Clinical and laboratory characteristics of patients with SCD were compared (to the entire EVOLVE cohort after removing those SCD cases) by using the $\chi^{2}$ test for categorical items and the 2 sample $t$ test for continuous items. After post hoc adjustment based on the 28 comparisons, a $p$ value of 0.0018 was re- 
Table 2. Gene level burden tests

\begin{tabular}{|c|c|c|c|c|}
\hline Gene symbol & Gene name/associated cardiac syndrome* & $\begin{array}{l}\text { Combined } \\
\text { variants, } n\end{array}$ & $\begin{array}{l}\text { Odds } \\
\text { ratios }^{+}\end{array}$ & $p$ value \\
\hline APOA4 & Apolipoprotein A4/FH & 8 & 0.40 & 0.006 \\
\hline$A P O E$ & Apolipoprotein E/FH & 2 & 0.12 & 0.017 \\
\hline$A B C C 9$ & ATP-binding cassette, subfamily C (CFTR/MRP), member 9/BrS & 3 & 4.51 & 0.020 \\
\hline LDLR & Low-density lipoprotein receptor/FH & 12 & 0.59 & 0.024 \\
\hline$T B X 3$ & T-box transcription factor TBX3/LQTS & 4 & 2.07 & 0.040 \\
\hline ANKRD1 & Ankyrin repeat domain 1 (cardiac muscle)/HCM & 5 & 0.23 & 0.043 \\
\hline HFE & Hemochromatosis/DCM & 5 & 1.71 & 0.045 \\
\hline ABCG5 & ATP-binding cassette (ABC) transporters G5/FH & 10 & 0.50 & 0.047 \\
\hline ZHX3 & Zinc fingers and homeoboxes protein 3/DCM & 10 & 0.60 & 0.054 \\
\hline GATAD1 & GATA zinc finger domain containing $1 / \mathrm{DCM}$ & 2 & 3.43 & 0.054 \\
\hline TXNRD2 & Thioredoxin reductase $2 / \mathrm{DCM}$ & 6 & 2.29 & 0.091 \\
\hline ZBTB17 & Zinc finger and BTB domain-containing protein 17/DCM & 2 & 0.31 & 0.101 \\
\hline HSPB8 & Heat shock protein $\mathrm{B} 8$ ( $\mathrm{aB}$-crystallin)/DCM & 2 & 6.08 & 0.111 \\
\hline$L P L$ & Lipoprotein lipase/FH & 7 & 0.46 & 0.114 \\
\hline$S G C B$ & Sarcoglycan, beta (43 kDa dystrophin-associated glycoprotein)/DCM & 2 & 0.10 & 0.120 \\
\hline KCND3 & Potassium voltage-gated channel, Shal-related subfamily, member 3/BrS & 4 & 0.24 & 0.125 \\
\hline$L T B P 2$ & Latent transforming growth factor beta binding protein $2 / \mathrm{MFS}$ & 21 & 0.59 & 0.133 \\
\hline KCNJ5 & Potassium inwardly rectifying channel, subfamily J, member 5/LQTS & 2 & 0.27 & 0.137 \\
\hline ELN & Elastin/AVD & 12 & 1.54 & 0.138 \\
\hline APOC2 & Apoliprotein $\mathrm{C} 2 / \mathrm{FH}$ & 6 & 3.10 & 0.144 \\
\hline NKX2-5 & NK2 homeobox 5/DCM & 2 & 0.43 & 0.154 \\
\hline CACNA2D1 & Calcium channel, voltage-dependent, alpha $2 /$ delta subunit $1 /$ SQTS & 4 & 1.61 & 0.158 \\
\hline SHOC2 & Soc-2 suppressor of clear homolog (C. elegans)/NS & 2 & 0.32 & 0.252 \\
\hline$S C N 4 B$ & Sodium channel, voltage-gated, type IV, beta/LQTS & 1 & 0.10 & 0.262 \\
\hline KCNE1 & Potassium voltage-gated channel, Isk-related family, member 1/LQTS & 1 & 0.32 & 0.262 \\
\hline PRKAG2 & Protein kinase, AMP-activated, gamma 2 noncatalytic subunit/HCM & 3 & 0.52 & 0.277 \\
\hline$M Y L 3$ & Myosin, light chain 3, alkali; ventricular, skeletal, slow/RCM & 1 & 0.12 & 0.279 \\
\hline$S C N 2 B$ & Sodium channel, voltage-gated, type II, beta subunit/BrS & 2 & 0.34 & 0.279 \\
\hline CACNA1C & Calcium channel, voltage-dependent, L type, alpha 1C subunit/LQTS & 11 & 1.62 & 0.285 \\
\hline TGFBR1 & Transforming growth factor, beta receptor 1/MFS & 1 & 0.11 & 0.288 \\
\hline ILK & Integrin-linked kinase/DCM & 1 & 0.13 & 0.298 \\
\hline FHL1 & Four and a half LIM domains $1 / \mathrm{HCM}$ & 1 & 0.13 & 0.298 \\
\hline $\mathrm{JPH} 2$ & Junctophilin 2/HCM & 6 & 1.52 & 0.298 \\
\hline MYH7 & Myosin, heavy chain 7 , cardiac muscle, beta/DCM & 4 & 1.96 & 0.300 \\
\hline$L M F 1$ & Lipase maturation factor $1 / \mathrm{FH}$ & 13 & 1.21 & 0.304 \\
\hline NOTCH1 & Notch 1/FAA & 24 & 0.78 & 0.317 \\
\hline SMAD4 & Small mothers against decapentaplegic (drospholia name), transcription factors/HCM & 1 & 7.90 & 0.317 \\
\hline ALMS1 & Centrosome- and basal body-associated protein/DCM & 44 & 1.18 & 0.319 \\
\hline CTF1 & Cardiotrophin 1/DCM & 1 & 2.80 & 0.323 \\
\hline GCKR & Glucokinase regulatory protein/FH & 7 & 0.53 & 0.341 \\
\hline SOS1 & Son of sevenless homolog 1 (Drosophila)/HCM & 4 & 0.51 & 0.342 \\
\hline$R A F 1$ & $\mathrm{v}$-raf-1 murine leukemia viral oncogene homolog $1 / \mathrm{HCM}$ & 1 & 6.37 & 0.349 \\
\hline
\end{tabular}

Sudden Cardiac Death in Patients on Dialysis
Cardiorenal Med 2021;11:174-183 DOI: $10.1159 / 000517123$ 
Table 2 (continued)

\begin{tabular}{|c|c|c|c|c|}
\hline Gene symbol & Gene name/associated cardiac syndrome* & $\begin{array}{l}\text { Combined } \\
\text { variants, } n\end{array}$ & $\begin{array}{l}\text { Odds } \\
\text { ratios }^{+}\end{array}$ & $p$ value \\
\hline$L A M A 4$ & Laminin, alpha 4/DCM & 11 & 1.39 & 0.358 \\
\hline SCN5A & Sodium channel, voltage-gated, type V, alpha subunit/ARVC & 15 & 0.76 & 0.359 \\
\hline SLC2A10 & Solute carrier family 2 (facilitated glucose transporter), member 10/FAA & 8 & 0.87 & 0.360 \\
\hline KRAS & v-Ki-ras2 Kirsten rat sarcoma viral oncogene homolog/NS & 1 & 6.33 & 0.363 \\
\hline CSRP3 & Cysteine and glycine-rich protein 3 (cardiac LIM protein)/HCM & 1 & 6.09 & 0.364 \\
\hline PTPN11 & Protein tyrosine phosphatase, nonreceptor type $11 / \mathrm{HCM}$ & 1 & 6.09 & 0.364 \\
\hline FKRP & Fukutin冈related protein (FKRP)/DCM & 6 & 0.60 & 0.366 \\
\hline KLF10 & Kruppel-like factor 10/HCM & 6 & 0.66 & 0.369 \\
\hline FBN1 & Fibrillin 1/MFS & 15 & 0.70 & 0.372 \\
\hline$C A L R 3$ & Calreticulin $3 / \mathrm{HCM}$ & 4 & 1.59 & 0.382 \\
\hline LAMA2 & Laminin, alpha $2 / \mathrm{HCM}$ & 42 & 0.89 & 0.386 \\
\hline$S G C D$ & Sarcoglycan, delta (35 kDa dystrophin-associated glycoprotein)/HCM & 2 & 1.56 & 0.388 \\
\hline TMEM43 & Transmembrane protein 43/ARVC & 4 & 1.42 & 0.397 \\
\hline COX15 & COX15 homolog, cytochrome c oxidase assembly protein (yeast)/HCM & 6 & 0.60 & 0.415 \\
\hline$M Y P N$ & Myopalladin/DCM & 15 & 1.14 & 0.433 \\
\hline KCNA5 & Potassium voltage-gated channel subfamily a member $5 /$ familial atrial fibrillation & 10 & 1.35 & 0.433 \\
\hline$T M P O$ & Thymopoietin/HCM & 13 & 0.88 & 0.457 \\
\hline SALL4 & Spalt-like transcription factor $4 /$ congenital heart disease & 9 & 0.72 & 0.461 \\
\hline TGFB2 & Transforming growth factor, beta $2 / \mathrm{FAA}$ & 3 & 0.42 & 0.462 \\
\hline PDLIM3 & PDZ and LIM domain 3/HCM & 5 & 0.77 & 0.465 \\
\hline SCN1B & Sodium channel subunit beta-1/BrS & 8 & 1.60 & 0.473 \\
\hline$T R D N$ & Triadin/CPVT & 11 & 0.87 & 0.489 \\
\hline$C B L$ & Cbl proto-oncogene, E3 ubiquitin protein ligase/NS & 3 & 0.45 & 0.490 \\
\hline KRT17 & Keratin $17 /$ possible arrythmias & 2 & 1.48 & 0.495 \\
\hline SREBF2 & Sterol regulatory element-binding transcription factor $2 / \mathrm{FH}$ & 10 & 1.21 & 0.513 \\
\hline$S G C G$ & Sarcoglycan gamma/DCM & 4 & 1.19 & 0.529 \\
\hline$B A G 3$ & BCL2-associated athanogene 3/DCM & 8 & 1.15 & 0.533 \\
\hline DOLK & Dolichol kinase/DCM & 6 & 1.66 & 0.538 \\
\hline$A B C G 8$ & ATP-binding cassette, subfamily G (WHITE), member 8/FH & 17 & 0.86 & 0.538 \\
\hline PRDM16 & PR domain containing $16 / \mathrm{HCM}$ & 14 & 1.29 & 0.558 \\
\hline DSP & Desmoplakin/ARVC & 23 & 0.89 & 0.564 \\
\hline$R Y R 2$ & Ryanodine receptor 2 (cardiac)/ARVC & 15 & 0.90 & 0.567 \\
\hline CACNB2 & Calcium channel, voltage-dependent, beta 2 subunit/BrS & 8 & 0.71 & 0.573 \\
\hline KCNE2 & Potassium voltage-gated channel, Isk-related family, member 2/LQTS & 3 & 0.68 & 0.573 \\
\hline$R B M 20$ & RNA-binding motif protein 20/DCM & 16 & 1.17 & 0.577 \\
\hline$A P O B$ & Apolipoprotein $\mathrm{B}$ (including $\mathrm{Ag}(\mathrm{x})$ antigen)/FH & 56 & 0.94 & 0.583 \\
\hline EYA4 & EYA transcriptional coactivator and phosphatase 4/DCM & 5 & 0.70 & 0.584 \\
\hline MYO6 & Myosin VI/HCM & 8 & 0.74 & 0.586 \\
\hline APOA5 & Apolipoprotein A5/FH & 3 & 1.25 & 0.604 \\
\hline FHL2 & Four and a half LIM domains $2 / \mathrm{HCM}$ & 3 & 0.70 & 0.607 \\
\hline GLA & Galactosidase, alpha/HCM & 4 & 1.43 & 0.608 \\
\hline$T T N$ & Titan/HCM & 437 & 1.01 & 0.608 \\
\hline CRELD1 & Cysteine rich with EGF-like domains 1/atrial septal defect & 4 & 0.84 & 0.609 \\
\hline$A K A P 9$ & A kinase (PRKA) anchor protein (yotiao) 9/LQTS & 30 & 0.91 & 0.617 \\
\hline DTNA & Dystrobrevin, alpha/LVNC & 5 & 1.42 & 0.619 \\
\hline MIB1 & Mindbomb E3 ubiquitin protein ligase 1/LVNC & 3 & 1.73 & 0.633 \\
\hline NEXN & Nexilin (F actin binding protein)/HCM & 7 & 0.88 & 0.634 \\
\hline TBX5 & T-box transcription factor 5/congenital heart disease and atrial fibrillation & 5 & 0.76 & 0.644 \\
\hline$S D H A$ & $\begin{array}{l}\text { Succinate dehydrogenase complex flavoprotein subunit A/rare cardiomyopathy and } \\
\text { pheochromocytoma }\end{array}$ & 13 & 0.92 & 0.645 \\
\hline$C R Y A B$ & Crystallin, alpha B/HCM & 2 & 1.71 & 0.652 \\
\hline GPIHBP1 & Glycosylphosphatidylinositol-anchored high-density lipoprotein-binding protein 1/FH & 3 & 1.19 & 0.653 \\
\hline DSC2 & Desmocollin $2 / \mathrm{HCM}$ & 11 & 0.88 & 0.659 \\
\hline KCNE3 & Potassium voltage-gated channel, Isk-related family, member 3/LQTS & 3 & 1.66 & 0.661 \\
\hline DPP6 & Dipeptidyl peptidase like 6/FH & 11 & 1.11 & 0.673 \\
\hline COL5A1 & Collagen type $\mathrm{V}$ alpha 1 chain/Ehlers-Danlos and cardiac repair & 20 & 0.89 & 0.677 \\
\hline CREB3L3 & CAMP responsive element-binding protein 3 like $3 / \mathrm{FH}$ & 3 & 1.62 & 0.680 \\
\hline
\end{tabular}


Table 2 (continued)

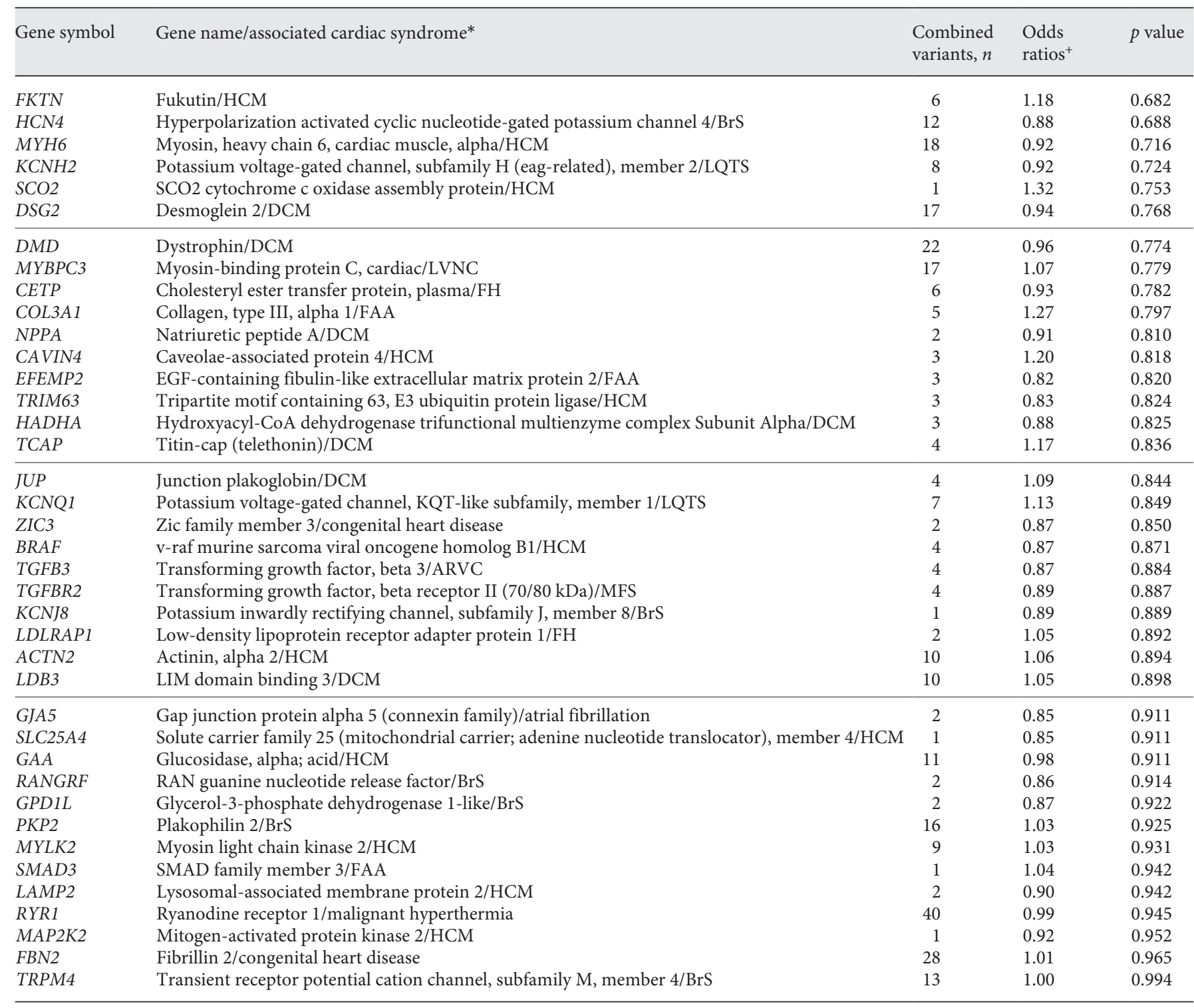

*Indicates the inherited cardiac condition identified with abnormalities in this gene. ARVC, arrhythmogenic right ventricular cardiomyopathy; AVD, aortic valve disease; BrS, Brugada syndrome; CPVT, catecholaminergic polymorphic ventricular tachycardia; DCM, dilated cardiomyopathy; FAA, familial aortic aneurysm; FH, familial hypercholesterolemia; HCM, hypertrophic cardiomyopathy; LQTS, long QT syndrome; LVNC, left ventricular noncompaction; MFS, Marfan syndrome; NS, Noonan syndrome; RCM, restrictive cardiomyopathy; SQTS, short QT syndrome. Additional cardiac phenotypes are written out. ${ }^{+} \mathrm{A}$ positive hazard ratio indicates increased positive association with the disease, and a negative hazard ratio indicates protective against the disease.

quired to indicate statistically significant difference in the 2 groups.

We conducted a gene-level burden test to identify genes that are associated with SCD risk. Different from traditional individual variant analysis, the gene-level burden test aggregates minor alleles for the variants that are in the same gene to improve statistical power and reduce multiple testing [21]. At each gene, minor allele carrier status of each individual is assigned based on the variants within the gene. For example, those with at least 1 minor allele are assigned as a carrier, while those who do not have any minor alleles across all the variants in the gene are assigned as a noncarrier. This status is then tested for association with the disease status such as SCD case-control status. An odds ratio $(\mathrm{OR})>1$ would indicate that having minor alleles in the gene increases the risk for SCD, while an $\mathrm{OR}<1$ would indicate that having minor alleles in the gene decreases the risk for SCD.

As the primary analysis, we conducted a gene-level burden test using the variants that passed filtering, as previously described. AfAn and EA samples were tested separately to reduce false discoveries due to population stratification that can arise when ana- 
Table 3. Disease burden test

\begin{tabular}{lrrll}
\hline Disease & $\begin{array}{l}\text { Genes, } \\
n\end{array}$ & $\begin{array}{c}\text { Combined } \\
\text { variants, } n\end{array}$ & $\begin{array}{c}\text { Odds } \\
\text { ratios }\end{array}$ & $p$ value \\
\hline $\begin{array}{l}\text { Inherited arrhythmias } \\
\text { LQTS }\end{array}$ & 16 & 137 & 0.87 & 0.084 \\
SQTS & 4 & 19 & 1.11 & 0.576 \\
BrS & 14 & 89 & 1.03 & 0.796 \\
CPVT & 6 & 29 & 0.84 & 0.177 \\
\hline Cardiomyopathies & & & & \\
HCM & 48 & 644 & 1.01 & 0.644 \\
DCM & 51 & 800 & 1.01 & 0.672 \\
ARVC & 12 & 556 & 1.00 & 0.803 \\
RCM & 9 & 33 & 1.13 & 0.355 \\
LVNC & 10 & 52 & 1.14 & 0.397 \\
NS & 9 & 16 & 0.73 & 0.435 \\
\hline Aortopathies & & & & \\
MFS & 4 & 41 & 0.64 & 0.073 \\
LDS & 3 & 20 & 0.70 & 0.300 \\
FAA & 12 & 92 & 1.03 & 0.694 \\
AVD & 3 & 51 & 0.96 & 0.832 \\
FH & 8 & 120 & 0.93 & 0.361 \\
\hline
\end{tabular}

LQTS, long QT syndrome; SQTS, short QT syndrome; BrS, Brugada syndrome; CPVT, catecholaminergic polymorphic ventricular tachycardia; HCM, hypertrophic cardiomyopathy; DCM, dilated cardiomyopathy; ARVC, arrhythmogenic right ventricular cardiomyopathy; RCM, restrictive cardiomyopathy; LVNC, left ventricular noncompaction; NS, Noonan syndrome; MFS, Marfan syndrome; LDS, Loeys-Dietz syndrome; FAA, familial aortic aneurysm; AVD, aortic valve disease; FH, familial hypercholesterolemia.

lyzed together. Using individual population analysis results, metaanalysis was conducted to identify genes that show a consistent effect in both populations. There were 1,526 variants available for testing after filtering for AfAa samples and 1,624 variants for EA samples. We used RVTESTS [22] and RAREMETAL [23] to conduct a gene-level burden test and meta-analysis. After adjusting for 153 genes, our Bonferroni-corrected statistical significance threshold was $3.3 \times 10^{-04}(0.05 / 153)$.

As a secondary analysis, we conducted a burden test at the disease level based on the assigned categories from the initial study involved in the TruSight development [15]. Using the same aggregation strategy as we have used for the primary gene-level analysis, we determined the minor allele carrier status for each individual for the variants in the genes associated with each of the 15 ICC. For example, LQTS had 16 genes including 137 variants. For diseaseburden testing, we assigned the minor allele carrier status for each individual based on the 137 variants that are in the 16 genes associated with LQTS. If a participant did not have any minor alleles across the 137 variants, then the participant was assigned as a noncarrier, while participants with at least 1 minor allele across the 137 variants were assigned as a carrier. Again, this carrier status was tested for association with the SCD status. Again, we tested the 2 (AfAn and EA) populations separately and then meta-analyzed to identify any consistent associations.

Last, we carried out a burden test at the gene functional level. Similar to the secondary burden test which was conducted at the disease level using the list of associated genes, we classified the genes into 11 functional categories based on GeneCards [24] reported gene function annotations.

To estimate the detectable ORs for gene-level burden testing in our dataset, we conducted a power calculation using GAS Power Calculator (http://csg.sph.umich.edu/abecasis/gas_power_calculator/index.html) [25]. We assumed a disease prevalence of $0.5 \%$ and combined a gene-level allele frequency of 5 and $10 \%$ (the proportion of samples with at least 1 minor allele for a gene). At $80 \%$ power, adjusted for multiple testing, this study was able to detect relative risks of 3.5 and 4.5 for a combined gene-level allele frequency of 10 and 5\%, respectively. For genes with a carrier frequency of $1 \%$ ( $1 \%$ of the samples are minor allele carriers), a relative risk of 10 could be detected at $70 \%$ power.

\section{Results}

Baseline demographics and laboratory values are shown in Table 1. There were no statistical differences among the demographics and laboratory values between those with SCD and those without SCD. Compared with the full EVOLVE cohort, patients with SCD were older $(p=$ $\left.1.7 \mathrm{e}^{-06}\right)$, more likely to have diabetes $\left(p=8.6 \mathrm{e}^{-05}\right)$, and have a history of any arrhythmia $\left(p=3.2 \mathrm{e}^{-4}\right)$. Nearly identical 
differences were observed when those tested in the current study were removed from the EVOLVE cohort. The burden test groups rare variants into a single variant for analyses, as detailed previously. Table 2 lists the gene name, its disease association, and number of variants examined in each gene. The gene with the largest number of variants is TTN (titin), but that is also the largest gene. After adjusting for the length of the captured region, the largest number of variants was found in $A P O C 2$, followed by KCNE3. Eleven genes showed a significant association between gene burden and SCD $(p<0.05)$ but were not statistically significant association after multiple testing corrections. All gene-level burden test results are shown in Table 2, and the specific references for each gene can be found in the supplemental table of [15]. To determine if genes relating to a specific cardiac disease (an inherited cardiac condition as denoted by the TruSight kit, e.g., dilated vs. hypertrophic cardiomyopathy) were more commonly associated with patients suffering SCD versus controls, we grouped genes by their associated disease and performed a burden test (Table 3). We did not observe any associations with $p$ values $<0.05$ in all 15 ICCs tested (Table 3). Last, to determine if a similar mechanism may be pathogenic in patients with SCD versus controls (e.g., potassium/sodium channel abnormalities), we grouped genes by their pathophysiology/ function as noted by the TruSight kit (Table 4). The burden testing at the gene function level also did not yield any associations with $p$ values $<0.05$ in all 11 functional categories tested with SCD. Overall, we observed no statistically significant associations between the rare variant burden at gene, associated disease, and gene function levels compared to SCD status in our EVOLVE trial.

\section{Discussion/Conclusion}

In the present study, we aimed to determine whether genes known to be associated with hereditary cardiovascular disease may be associated with adjudicated SCD events in patients with ESKD from the EVOLVE trial. No significant associations were observed, although our power was limited. Despite strong evidence of heritability with cardiac arrest based on CMS data [8], we did not see significant associations. A previous study in patients with ESKD [9] examined 24 genes in 47 patients and 38 with long QTc present on electrocardiogram characterized as abnormal before, after, or before and after dialysis. Two of the 5 patients with potassium channel defects $(\mathrm{KCNH} 2$ and KCNE1) subsequently died of SCD. These same 24 genes were examined in the present study, but we were

Sudden Cardiac Death in Patients on Dialysis
Table 4. Gene function burden test

\begin{tabular}{lccl}
\hline Gene function grouping & $\begin{array}{c}\text { Combined } \\
\text { variants, } n\end{array}$ & $\begin{array}{l}\text { Odds } \\
\text { ratio }\end{array}$ & $\begin{array}{l}p \\
\text { value }\end{array}$ \\
\hline Adaptor signal transduction & 15 & 1.20 & 0.395 \\
Development gene expression & 133 & 0.99 & 0.948 \\
Ion channel & 264 & 0.94 & 0.278 \\
Lipid metabolism & 133 & 0.93 & 0.346 \\
Metabolism & 53 & 1.00 & 0.998 \\
Mitochondria & 25 & 0.85 & 0.295 \\
Signaling RAS/MAPK & 18 & 0.81 & 0.566 \\
Transport & 46 & 1.00 & 0.994 \\
Vascular connective tissue & 123 & 0.91 & 0.411 \\
Cytoskeleton & 853 & 1.01 & 0.530 \\
Signal transduction & 161 & 0.92 & 0.274 \\
\hline
\end{tabular}

unable to identify association between $\mathrm{KCNH} 2$ and $K C N E 1$ and SCD in our samples $(p=0.72$ and 0.26 , respectively). To our knowledge, these are the only 2 studies to examine potential genetic risks in patients with ESKD and SCD. Our study of 126 patients with SCD, though much larger than Coll et al. [26], is still underpowered to detect rare variants. Thus, the overall results of this modest-sized study suggest that known genes associated with hereditary genetic diseases in the general population do not explain the high rates of SCD in patients undergoing dialysis. Our previous work did however find associations of SNPs in the ACE gene with decreased risk of SCD in EA and another SNP with increased risk of SCD in AfAn [10].

There are many risk factors for SCD in patients with ESKD. First, our study showed that compared to the overall EVOLVE cohort, patients with SCD were more likely to be older, have diabetes, and with a previous history of arrhythmia. Second, left ventricular hypertrophy, present in $80 \%$ of patients starting dialysis, increases the risk [27, 28]. Third, obstructive sleep apnea is common in patients undergoing dialysis [29] and is associated with a higher risk of SCD after adjusting for demographics (HR 3.28 [95\% CI 1.12-9.57]) [29]. Fourth, 70\% of patients starting dialysis have significant coronary artery calcification [30], which has been shown to predict SCD and Framingham risk factors in the general population [31]. Finally, disordered fluid and electrolyte fluxes with dialysis may still be causative, although we did not find any differences in serum electrolytes between those with and without SCD in this study, and Pun et al. [32], analyzing the entire cohort in the EVOLVE study, found no relationship between dialysate calcium concentration and cardiovascular events, including SCD.

Unfortunately, we still lack a complete understanding of the pathophysiology of SCD, making treatment and/or 
prevention difficult. In the general population, implantable defibrillators are the treatment of choice, especially with reduced ejection fraction. A meta-analysis demonstrated that implantable defibrillators reduced mortality in non-dialysis-requiring CKD but not in patients undergoing dialysis [33]. The ICD-2 trial confirmed a lack of efficacy in 188 patients with ESKD and an ejection fraction $>35 \%$ [34]. The type of fatal arrhythmia in ESKD may be different from the general population with SCD. Five studies have utilized implantable loop records during and between dialysis sessions, with a follow-up from 6 to 21 months [33-37]. All of these studies noted a high rate of both atrial fibrillation and bradycardia, the latter affecting $25-30 \%$ of patients with only a minority of patients suffering from ventricular arrhythmias. Three studies found increased arrhythmias during the long (3-day) versus 2-day interval between hemodialysis sessions, suggesting the magnitude of volume retention (or accumulation of uremic toxins) may play a role $[33,36,37]$. In the general population, the treatment of nonatherosclerotic SCD similarly remains unclear [35], although genetic testing is suggested in patients with a family history of an early cardiac event. Based on the current study, we cannot recommend such an approach in patients with ESKD.

This study has several limitations, most importantly, sample size, although it remains the largest study to date. The modest sample size severely limited the statistical power of the study, only allowing identification of association with genes having large relative risks $(R R>3.5)$. The sample tested may not be generalizable to the hemodialysis population at large, as $70 \%$ of the patients were on dialysis for $>2$ years since all had moderate to severe hyperparathyroidism. It is conceivable that individuals with rare genetic variants may have died earlier after dialysis initiation. Despite these limitations, the study tested the most likely rare ge- netic variants and, importantly, utilized endpoints that were adjudicated by an independent committee.

In conclusion, we examined rare cardiovascular gene variants in a case-control study of prevalent patients on hemodialysis, matched for age, sex, diabetes, and duration of dialysis. None of the tested genes had different burden for rare function variants in patients who suffered from SCD compared to the matched controls. These data do not support genetic testing to stratify patients on dialysis for risk of SCD.

\section{Statement of Ethics}

This was a substudy of a clinical trial (EVOLVE, NCT00345839) using de-identified DNA samples and was considered "not subject to common rule" per our Institutional Review Board.

\section{Conflict of Interest Statement}

Dr. Moe and Dr. Chertow served in the Academic Executive Committee and Publication Committee for the EVOLVE trial. Dr. Chertow has received funding from Amgen to conduct secondary analyses of the EVOLVE trial. Dr. Vatta receives salary and stocks from Invitae Corporation.

\section{Funding Sources}

This study was funded by the National Institute of Health (R01DK100306). The parent study EVOLVE was funded by Amgen.

\section{Author Contributions}

The project was conceived and funding obtained by S.M.M. and G.M.C. T.-H.S.-A., M.V., M.A., and L.W. conducted the analyses. All the authors worked together to interpret the results. T.-H.S.-A. and S.M.M. drafted the manuscript, and all the authors approved the final submission.

\section{References}

1 Baigent C, Landray M, Leaper C, Altmann P, Armitage J, Baxter A, et al. First United Kingdom heart and renal protection (UK-HARPI) study: biochemical efficacy and safety of simvastatin and safety of low-dose aspirin in chronic kidney disease. Am J Kidney Dis. 2005 Mar;45(3):473-84.

2 Landray M, Baigent C, Leaper C, Adu D, Altmann P, Armitage J, et al. The second United Kingdom heart and renal protection (UKHARP-II) study: a randomized controlled study of the biochemical safety and efficacy of adding ezetimibe to simvastatin as initial therapy among patients with CKD. Am J Kidney Dis. 2006 Mar;47(3):385-95.
3 Herzog CA, Asinger RW, Berger AK, Charytan DM, Díez J, Hart RG, et al. Cardiovascular disease in chronic kidney disease. A clinical update from kidney disease: improving global outcomes (KDIGO). Kidney Int. 2011 Sep; 80(6):572-86

4 Wheeler DC, London GM, Parfrey PS, Block GA, Correa-Rotter R, Dehmel B, et al. Effects of cinacalcet on atherosclerotic and nonatherosclerotic cardiovascular events in patients receiving hemodialysis: the evaluation of cinacalcet $\mathrm{HCl}$ therapy to lower cardioVascular events (EVOLVE) trial. J Am Heart Assoc. 2014 Nov 17;3(6):e001363.
5 Herzog CA. Can we prevent sudden cardiac death in dialysis patients? Clin J Am Soc Nephrol. 2007 May;2(3):410-2.

6 Alqahtani F, Almustafa A, Shah K, Akram Y, Abbasi D, Rattan R, et al. Sudden cardiac arrest in end-stage renal disease patients on dialysis: a nationwide study. Pacing Clin Electrophysiol. 2018 Nov;41(11):1467-75.

7 Pun PH. The interplay between CKD, sudden cardiac death, and ventricular arrhythmias. Adv Chronic Kidney Dis. 2014 Nov;21(6):480-8.

8 Chan KE, Newton-Cheh C, Gusella JF, Maddux FW. Heritability of risk for sudden cardiac arrest in ESRD. J Am Soc Nephrol. 2015 Nov;26(11):2815-20. 
9 Coll M, Ferrer-Costa C, Pich S, Allegue C, Rodrigo E, Fernández-Fresnedo G, et al. Role of genetic and electrolyte abnormalities in prolonged QTc interval and sudden cardiac death in end-stage renal disease patients. PLoS One. 2018;13(7): 0200756.

10 Moe SM, Long J, Schwantes-An TL, Decker BS, Wetherill L, Edenberg HJ, et al. Angiotensin-related genetic determinants of cardiovascular disease in patients undergoing hemodialysis. Nephrol Dial Transplant. 2019 Nov;34(11):1924-31.

11 Bezzina CR, Lahrouchi N, Priori SG. Genetics of sudden cardiac death. Circ Res. 2015 Jun 5; 116(12):1919-36

12 Chertow GM, Correa-Rotter R, Block GA, Drueke TB, Floege J, Goodman WG, et al. Baseline characteristics of subjects enrolled in the evaluation of cinacalcet $\mathrm{HCl}$ therapy to lower cardiovascular events (EVOLVE) trial. Nephrol Dial Transplant. 2012 Jul;27(7): 2872-9.

13 Investigators ET, Chertow GM, Block GA Correa-Rotter R, Drüeke TB, Floege J, et al. Effect of cinacalcet on cardiovascular disease in patients undergoing dialysis. $\mathrm{N}$ Engl J Med. 2012 Dec 27;367(26):2482-94

14 Moe SM, Wetherill L, Decker BS, Lai D, Abdalla S, Long J, et al. Calcium-sensing receptor genotype and response to cinacalcet in patients undergoing hemodialysis. Clin J Am Soc Nephrol. 2017 Jul 7;12(7):1128-38.

15 Pua CJ, Bhalshankar J, Miao K, Walsh R, John S, Lim SQ, et al. Development of a comprehensive sequencing assay for inherited cardiac condition genes. J Cardiovasc Transl Res. 2016 Feb;9(1):3-11.

16 McKenna A, Hanna M, Banks E, Sivachenko A, Cibulskis K, Kernytsky A, et al. The genome analysis toolkit: a mapReduce framework for analyzing next-generation DNA sequencing data. Genome Res. 2010 Sep;20(9): 1297-303.

17 Van der Auwera GA, Carneiro MO, Hartl C, Poplin R, Del Angel G, Levy-Moonshine A, et al. From FastQ data to high confidence variant calls: the genome analysis toolkit best practices pipeline. Curr Protoc Bioinformatics. 2013;43:11.10.1-11.10.33.
18 Li H, Durbin R. Fast and accurate short read alignment with Burrows-Wheeler transform. Bioinformatics. 2009 Jul 15;25(14):1754-60.

19 Wang K, Li M, Hakonarson H. ANNOVAR: functional annotation of genetic variants from high-throughput sequencing data. $\mathrm{Nu}-$ cleic Acids Res. 2010 Sep;38(16):e164.

20 Karczewski KJ, Francioli LC, Tiac G, Cummings BB, Alfoldi J, Wang Q, et al. Variation across 141,456 human exomes and genomes reveals the spectrum of loss-of-function intolerance across human protein-coding genes. biorxiv. 2019.

21 Povysil G, Petrovski S, Hostyk J, Aggarwal V, Allen AS, Goldstein DB. Rare-variant collapsing analyses for complex traits: guidelines and applications. Nat Rev Genet. 2019 Dec 1; 20(12):747-59.

22 Zhan X, Hu Y, Li B, Abecasis GR, Liu DJ. RVTESTS: an efficient and comprehensive tool for rare variant association analysis using sequence data. Bioinformatics. 2016 May 1; 32(9):1423-6.

23 Feng S, Liu D, Zhan X, Wing MK, Abecasis GR. RAREMETAL: fast and powerful metaanalysis for rare variants. Bioinformatics. 2014 Oct;30(19):2828-9.

24 Stelzer G, Rosen N, Plaschkes I, Zimmerman S, Twik M, Fishilevich S, et al. The geneCards suite: from gene data mining to disease genome sequence analyses. Curr Protoc Bioinformatics. 2016 Jun 20;54:1.30.1-1.30.33.

25 Skol AD, Scott LJ, Abecasis GR, Boehnke M. Joint analysis is more efficient than replication-based analysis for two-stage genomewide association studies. Nat Genet. 2006 Feb; 38(2):209-13

26 Coll B, Betriu A, Martinez-Alonso M, Borras M, Craver L, Amoedo ML, et al. Cardiovascular risk factors underestimate atherosclerotic burden in chronic kidney disease: usefulness of non-invasive tests in cardiovascular assessment. Nephrol Dial Transplant. 2010 Mar 17; 25(9):3017-25.

27 Spirito P, Maron BJ. Relation between extent of left ventricular hypertrophy and occurrence of sudden cardiac death in hypertrophic cardiomyopathy. J Am Coll Cardiol. 1990 Jun; 15(7):1521-6

28 Zoccali C, Benedetto FA, Mallamaci F, Tripepi G, Giacone G, Stancanelli B, et al. Left ventricular mass monitoring in the follow-up of dialysis patients: prognostic value of left ventricular hypertrophy progression. Kidney Int. 2004 Apr;65(4):1492-8.
29 Kerns ES, Kim ED, Meoni LA, Sozio SM, Jaar BG, Estrella MM, et al. Obstructive sleep apnea increases sudden cardiac death in incident hemodialysis patients. Am J Nephrol. 2018;48(2):147-56.

30 Block GA, Spiegel DM, Ehrlich J, Mehta R, Lindbergh J, Dreisbach A, et al. Effects of sevelamer and calcium on coronary artery calcification in patients new to hemodialysis. Kidney Int. 2005 Oct;68(4):1815-24.

31 Taylor AJ, Burke AP, O’Malley PG, Farb A, Malcom GT, Smialek J, et al. A comparison of the Framingham risk index, coronary artery calcification, and culprit plaque morphology in sudden cardiac death. Circulation. 2000 Mar 21;101(11):1243-8.

32 Pun PH, Abdalla S, Block GA, Chertow GM, Correa-Rotter R, Dehmel B, et al. Cinacalcet, dialysate calcium concentration, and cardiovascular events in the EVOLVE trial. Hemodial Int. 2016 Jul;20(3):421-31.

33 Fu L, Zhou Q, Zhu W, Lin H, Ding Y, Shen Y, et al. Do implantable cardioverter defibrillators reduce mortality in patients with chronic kidney disease at all stages? Int Heart J. 2017 May 31;58(3):371-7.

34 Jukema JW, Timal RJ, Rotmans JI, Hensen LCR, Buiten MS, de Bie MK, et al. Prophylactic use of implantable cardioverter-defibrillators in the prevention of sudden cardiac death in dialysis patients. Circulation. 2019 Jun 4, 139(23):2628-38

35 Kadakia RS, Link MS, Dominic P, Morin DP. Sudden cardiac death in nonischemic cardiomyopathy. Prog Cardiovasc Dis. 2019 MayJun;62(3):235-41.

36 Sacher F, Jesel L, Borni-Duval C, De Precigout V, Lavainne F, Bourdenx JP, et al. Cardiac Rhythm Disturbances in Hemodialysis $\mathrm{Pa}$ tients: Early Detection Using an Implantable Loop Recorder and Correlation With Biological and Dialysis Parameters. JACC Clin Electrophysiol. 2018;4(3):397-408

37 Roy-Chaudhury P, Tumlin JA, Koplan BA, Costea AI, Kher V, Williamson D, et al. Primary outcomes of the Monitoring in Dialysis Study indicate that clinically significant arrhythmias are common in hemodialysis patients and related to dialytic cycle. Kidney Int. 2018;93(4):941-51.
Sudden Cardiac Death in Patients on Dialysis
Cardiorenal Med 2021;11:174-183 